\title{
GLICOALCALOIDES: TOXINAS NATURALES EN LOS ALIMENTOS VEGETALES
}

Guillermo Bornaz Acosta

\section{INTRODUCCION}

Los glicoalcaloides, un tipo de toxinas familiares que se encuentran naturalmente en los vegetales, fueron descubiertos accidentalmente, cuando un grupo de investigadores determinaba el grado de contaminación que se producía al tratar una planta con piretroides (insecticida que inhibela acetil colinesterasa de insectos). Fue sorprendente ver que las plantas no tratadas con este tóxico, también presentaban capacidad de inhibir este tipo de enzima (BUSWAY y col 1987).

En la década de los treinta se demostró que un extracto de tomate presentaba efectos tóxicos sobre Fusarium oxiporum f. Ivcopersici, un hongo patógeno del tomate. El principio activo fue aislado yle denominaron "Iycopersicina" (JADHAV, 1981); posteriormente los mismos autores cambiaron la designación desde fungistático a "tomatina" para evitar la confusión con el "lycopeno", pigmento rojo del tomate. El término "tomatina" o "alfa-tomatina" se utiliza desde entonces para describir al alcaloide en su forma pura.

Estas sustancias, frecuentemente, se encuentran en diversos vegetales, en especial en la familia Solaneceae. Los integrantes de esta familia contienen numerosos glicoalcaloides; sustancias alcaloides con nitrógeno en su estructura esteroide, que generalmente se encuentran unidos a una o más moléculas de azúcar, que toman el nombre de AGLICONAS cuando las pierden. El tubérculo dela papa (Solanun tuberosum), como una de las especies más representativa delas solanaceas, presentalos siguientes glicoalcaloides:

\begin{tabular}{|c|c|c|}
\hline AGLICONA & GLICOALCALOIDE & CARBOHIDRATO \\
\hline Solanidin & $\begin{array}{l}\text { Alfa-solanina } \\
\text { Alfa-chaconina } \\
\text { Dehidrocommersenin }\end{array}$ & $\begin{array}{l}\text { Solatriosa } \\
\text { Chacotriosa } \\
\text { Commertetrosa }\end{array}$ \\
\hline Demiseidina & $\begin{array}{l}\text { Demissina } \\
\text { Commersonina }\end{array}$ & $\begin{array}{l}\text { Licotetrosa } \\
\text { Commertetrosa }\end{array}$ \\
\hline Acetilpeptidina & $\begin{array}{l}\text { Leptina I } \\
\text { Leptina II }\end{array}$ & $\begin{array}{l}\text { Chacotriosa } \\
\text { Solatriosa }\end{array}$ \\
\hline Tomatidenol & $\begin{array}{l}\text { Alfa-solamarina } \\
\text { Beta-solamarina }\end{array}$ & $\begin{array}{l}\text { Solatriosa } \\
\text { Chacotriosa }\end{array}$ \\
\hline Tomatidina & Alfa-tomatina & Licotetrosa \\
\hline
\end{tabular}

Fuente: Wolfe, 1987

\section{ESTRUCTURA QUIMICA DE LOS GLICOALCA- LOIDES}

Los alcaloides son una variedad de estructuras químicas que van desde simples monociclos, como la piperidina y coniina de la cicuta, hasta hexa y hepta cíclicos, como la solanidina, tomatidina y estricnina. Todos presentan un nitrógeno orgánico en su estructura. Existen en la naturaleza por lo menos 5500 alcaloides caracterizados.

Los alcaloides presentes en la papa poseen un núcleo esteroide con un nitrógeno incorporado en la molécula por ciclización de la cadena lateral esteroidal . La mayoría se presentan asociados a carbohidratos, como glicoalcaloides, que al separar el componente glucídico, quedan transformados en agliconas que guardan mucha semejanza con el colesterol (JADHAV, 1981).

\section{FUNCIONES DE LOS GLICOALCALOIDES}

La alfa-tomatina se sintetiza en la planta cuando es infectada por el hongo Fusarium oxvsporum $\mathbf{f}$. lycopersici; lo que provoca una inhibición fungal. No se dispone de literatura, si la injuria mecánica afecta al 
metabolismo de la alfa-tomatina.

Frecuentemente los alcaloides desempeñan un papel de repelentes alimentarios. Existen pruebas de que un pequeño número de plantas usan alcaloides como mecanismo de resistencia contra las enfermedades microbianas. Más interesante es el hecho de que ciertos animales pueden imitar a las plantas, al sintetizar sus propios alcaloides como mecanismos de defensa, como ocurre en las hormigas, en algunos organismos marinos y hasta en algunos anfibios. Esto demuestra que la capacidad para sintetizar alcaloides, no es exclusividad de las plantas.

\section{FARMACOLOGIADELOS GLICOALCALOIDES}

El consumo habitual de los glicoalcaloides, en especial de los presentes en la papa, ha impulsado el estudio de la influencia de estos compuestos sobre el metabolismo. Diversos estudios alimentarios han sugerido que la alfa-tomatina de la dieta, forma un complejo no absorbiblecon el colesterol a nivel intestinal, no afectando la absorción de los ácidos biliares. En este estudio, la tomatina causó incremento de la tasa de síntesis de colesterol hepático e intestinal, y un aparente incremento en la excreción de este esteroide sin afectar la excreción de los ácidos biliares.

En un reciente estudio realizado en el laboratorio de Bioquímica de la UNSA, se encontró que ratas alimentadas con una dieta a base de papa durante 10 días, presentan una disminución significativa de los niveles totales de colesterol sanguíneo, los mismos que tienden a recuperarse en 10 días, después de haberles suprimido dicha alimentación. Los niveles de HDLcolesterol tienden a incrementarse sin ser esto significativo (Castillo, 1991).

Diversos glicoalcaloides que se encuentran en la papa, producen efectos cardiatónicos, similares a los producidos por los glicósidos cardíacos. Los efectos inotrópicos de tomatina $(10 \mathrm{ug} / \mathrm{ml})$ fueron similares a los dealfa-solanina y alfa-chaconina, cuando se estudiaron atemperatura ambiente sobrelapreparación deventrículo aislado. La potencia de los glicoalcaloides estudiados fueligeramente menor que el compuesto katrofantósido, pero la tomatina iue la más potente

Estos alcaloides presentan una marcada capacidad inhibitoria de la acetilcolinesterasa, tanto en humanos como en animales. Esta actividad es, probablemente, una función del núcleo esteroide y del grupo amino, los cuales actuarían como un sitio de unión a la enzima, provocando una inhibición del tipo no competitiva. Adicionalmente se ha demostrado actividades antihistamínicas y citostáticas de la tomatina 98
(JADHAV, 1981). Demostrándose quela inyección intraperitoneal de extractos vegetales en cobayos, protegían a los animales de los efectos letales de un aerosol de histamina.

La actividad anti-inflamatoria de la tomatina ha sido evaluada por diversosmétodos. Latomatina demostró inhibición de edema experimental, al ser administrada por vía oral o intramuscular a ratas. Esta inhibición fue mayor en ratas adrenalectomizadas que en animales intactos. La administración subcutánea, durante siete días a animales intactos, disminuyó el crecimiento del tejido de granulación, siendo este efecto similar al de los esteroides.

Adicionmalmentea la actividad fungistática de la tomatina y otros glicoalcaloides, se ha demostrado que también poseen actividad antibiótica, inhibiendo efectivamenteel crecimientodediversosmicroorganismos, excepto E. Coli y Penicillium notatum. Estos resultados indican la posibilidad del uso terapéutico en ciertas infecciones en humanos.

Laactividad antifúngica de la tomatina, al parecer se encuentra en la porción tomatidina de la molécula. Por el contrario, los productos de la hidrólisis de la tomatina parecen tener menos eficacia que la tomatina pura (ALLEN, 1968). El modo de acción de la tomatina no se conoce completamente. La evidencia acumulada indica que la tomatina o algún producto de hidrólisis, es capaz de inhibir la acción de la deshidrogenasa málica en E.coli.

Adicionalmente se ha postulado un mecanismo de acción antifúngica, mediado a través de la capacidad unidora de colesterol por la tomatina. Hongos que carecen de colesterol en sus membranas, son insensibles a la tomatina; este glicoalcaloide podría alterar la permeabilidad de la membrana y subsecuentemente destruir la membrana celular.

Esta hipótesis es consistente con la mayor toxicidad de la tomatina, en relación a los glicoalcaloides de la papa. Sin embargo no se descarta la posibilidad de otros mecanismos responsables de las acciones tóxicas de la tomatina.

Los glicoalcaloides de la papa son tóxicos para los seres humanos bajo ciertas condiciones. Se ha reportado que el consumo accidental de papas con un alto contenido de glicoalcaloides, puede provocar severos malestares y en algunos casos la muerte.

HARRIS y COCKBURN examinaros 61 casos 
de envenenamiento en Escocia, uno de los cuales fue fatal.

El análisis de las papas que produjeron el envenenamiento, reveló la existencia de $410 \mathrm{mg}$. de glicoalcaloides de $1 \mathrm{Kg}$. de papa. Asímismo reportaron que 9 miembros de una familia de Illinois (EE.UU) que comieron papas verdosas, mostraron algunos signos de intoxicación (HADHAV, 1981). Yen Canadá, se demostró que las papas de sabor amargo, causaban molestias a consumidores y cultivadores, como consecuencia de su lto contenido en glicoalcaloides.

Los síntomas que producen los glicoalcaloides son, generalmente, los de una molestia gastrointestinal aguda con dolor abdominal, vómitos y diarrea.

En seres humanos, son capaces de generar efectos teratógenos, que incluyen lesiones en el esqueleto, como en el caso conocido como espina bífida. Esto, a consecuencia de un excesivo consumo de papas, que contenían el alcaloide solanina, por mujeres en estado de gestación. Ental sentido, RENWICKadelantó unateoría, según la cual los defectos de nacimiento, la anencefalia y la espina bífida quística, podrían ser resultado de la ingesta depapa, con elevado contenido de glicoalcaloides, por mujeres embarazadas. La relación entre este defecto congénito y la dieta es, sin embargo complejo, y la implicación de los componentes de la papa, como agentes causales, no está bien probada (KUC, 1975).

\section{ASPECTOS BIOQUIMICOS DE LOS GLICOAL- CALOIDES}

La Tomatidina y la solanidina representan sapogeninas en las que el oxígeno del anillo, ha sido reemplazado por un grupo amino secundario. Se sabe que solasodina deriva deuna ruta acetatomevalonato que también compromete al escualeno y colesterol, como intermediarios claves. Así, aunque la ruta biosintética para tomatidina no se conoce con exactitud, puede inferirse sobre la base de similitud estructural un compromiso con rutas de alcaloides esteroides.

Labiosíntesis detomatidina y de alfa-tomatidina incorporauna marca radioactiva deacetato, mevalonato, cicloartenol, lanosterol y colesterol en la porción esteroidal delalcaloide. Un ataque electrolítico por un catión hidroxilo en la posición C-3, media la ciclización de escualeno y es seguido de una serie de modificaciones moleculares, conducentes a la formación de colesterol.

En un intento por investigar la introducción denitrógeno en los alcaloides esteroidales, RONCHETTI y col. (1975) demostraron que el nitrógeno debe introducirse en la posición C-26 del colesterol, antes de la formación del anillo tetrahidrofurano. Se tiene poca información sobre la glicosilación enzimática de la tomatina, una aglicona hidrofóbica, que constituye, al menos parcialmente, un proceso desolubilización, debido a que alfa-tomatina se encuentra principalmente en la porción soluble de la célula.

El principal sitio de degradación de alfa-tomatina es en la fruta del tomate. /Se considera que la biodegradación ocurreen el estado deaglicona, posterior a una deshidrogenación. Es interesante señalar que los tomates son incapaces de degradar alfa-tomatina y la enzima responsable es activada o sintetizada durante la maduración de la fruta. No se tiene información sobre las características del sistema enzimático que causa la degradación de alfa-tomatina en tomates blandos.

\section{DISTRIBUCION DE LOS ALCALOIDES EN LAS PLANTAS}

Existe información limitada sobre la distribución relativa devarios glicoalcaloides, pero se tiene evidencias que, en los tejidos meristemáticos, presentan un alto contenido.

Muchos estudios han mostrado que los glicoalcaloides son formados en las células del parénquima, peridermo y corteza de los tubérculos, y en áreas de alta actividad metabólica, como las yemas del tubérculo. Además, se ha demostrado que en las hojas y en los tubérculos, se produce una disminución en el contenido de glicoalcaloides durante el proceso de vegetación.

La tomatina está presente en el tomate y se encuentra también en todos los órganos de la planta. La mayor concentración de este alcaloide se encuentra en las flores, en especial en los ovarios polinizados.

\section{PERSPECTIVAS}

El rol fisiológico y bioquímico de los glicoalcaloides en animales y el hombre estálejos de ser comprendido.

La inhibición dela ecetil colinesterasa en insectos y el hombre, por estas sustancias, recientemente está recibiendo gran atención; por lo que será importante estudiar en primer lugar, el efecto de los glicoalcaloides dela papay el tomate sobre el rendimiento académico de estudiantes de diferentes niveles, alimentos que con muchafrecuencia son consumidos por nuestrapoblación. En segundo lugar, estudiar la factibilidad de aislar industrialmente glicoalcaloides producidos por biotecnología, con la finalidad de utilizarlos como insecticidas naturales en el combate de determinadas plagas agrícolas. 


\section{BIBUOGRAFIA}

Allen EH, Kuc K. (1968) Alpha-solanine and alphachaconone as Fungotoxic compounds in extracts of Irish potato tubers.

Busway RJ, Savage SA, Fegunson BS (1987) Inhibi tion of acetylcholinestesase by solanaceaus glycoalcaloids and alcaloids. Am J Potato

Castillo LR y col (1991) Medida de la actividad colinesterásica y los niveles de colesterol y HDI colesterol en plasma sanguíneo de rutas alimentadas con papa. XVII Congreso Peruano de Química.

Cheeke PR (1989) Toxicants of plants origin. CRC Press Inc. Boca Raton: Florida.

Jadhav SJ, Sharma P, Salunke DK (198i) Naturally occuring toxic alcaloids in food. CRC critical

\section{Rev toxic}

Kuk J (1975) Teretogenic constituents of potatoes. Recent Adv Pithochen

Kusano G, et al (1987) Solanun alkaloids as inhibitors of enzimatic conversion of dihidrolanosterol into cholesterol. Chem Pharm bull.

Reeve RM, Hantala E, Weavere ML (1969) Anatomy an compositional varation Within Potatoes II Phenolies enzimas and other minor compo nents. Am Potato J.

Renwich JH y col. (1984) Neural tuber defects produced in Siriam hamsters glycoalcaloids by potato glycoalcaloids Teratology

Wolfe JA. (1987) The potato in the human diet. Cam bridge University Press: Cambridge.
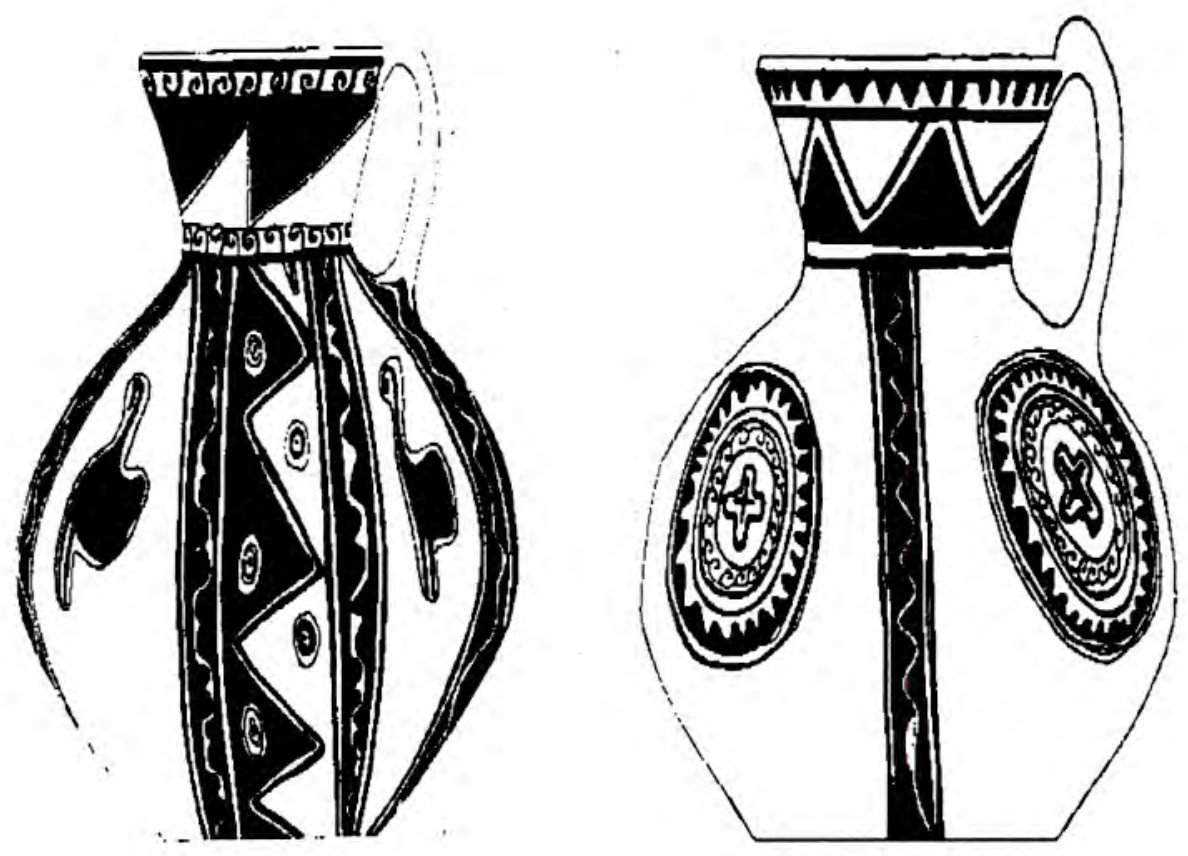